\title{
Optimizing Day-Ahead Electricity Market Prices: Increasing the Total Surplus for Energy Exchange Istanbul
}

\author{
Kursad Derinkuyu, ${ }^{\text {a,b }}$ Fehmi Tanrisever, ${ }^{\mathrm{c}}$ Nermin Kurt, ${ }^{\mathrm{b}}$ Gokhan Ceyhan ${ }^{\mathrm{b}}$ \\ ${ }^{\mathbf{a}}$ Department of Industrial Engineering, TOBB University of Economics and Technology, 06510 Ankara, Turkey; ${ }^{\mathbf{b}}$ Optimization and \\ Algorithms Division, Energy Exchange Istanbul, 34394 Istanbul, Turkey; ${ }^{\mathbf{c}}$ Faculty of Business Administration, Bilkent University, \\ 06800 Ankara, Turkey \\ Contact: kderinkuyu@etu.edu.tr, (D) https://orcid.org/0000-0002-4065-8857 (KD); tanrisever@bilkent.edu.tr, \\ (D) https:// orcid.org/0000-0002-3921-3877 (FT); nermin.kurt@epias.com.tr (NK); gokhan.ceyhan@epias.com.tr (GC)
}

Received: October 30, 2017

Revised: August 2, 2018; October 27, 2018

Accepted: October 30, 2018

Published Online in Articles in Advance: November 5, 2019

https://doi.org/10.1287/msom.2018.0767

Copyright: @ 2019 INFORMS

\begin{abstract}
Problem definition: We design a combinatorial auction to clear the Turkish dayahead electricity market, and we develop effective tabu search and genetic algorithms to solve the problem of matching bidders and maximizing social welfare within a reasonable amount of time for practical purposes. Academiclpractical relevance: A double-sided blind combinatorial auction is used to determine electricity prices for day-ahead markets in Europe. Considering the integer requirements associated with market participants' bids and the nonlinear social welfare objective, a complicated problem arises. In Turkey, the total number of bids reaches 15,000, and this large problem needs to be solved within minutes every day. Given the practical time limit, solving this problem with standard optimization packages is not guaranteed, and therefore, heuristic algorithms are needed to quickly obtain a high-quality solution. Methodology: We use nonlinear mixed-integer programming and tabu search and genetic algorithms. We analyze the performance of our algorithms by comparing them with solutions commercially available to the market operator. Results: We provide structural results to reduce the problem size and then develop customized heuristics by exploiting the problem structure in the day-ahead market. Our algorithms are guaranteed to generate a feasible solution, and Energy Exchange Istanbul has been using them since June 2016, increasing its surplus by 448,418 Turkish liras (US\$128,119) per day and 163,672,570 Turkish liras (US\$46,763,591) per year, on average. We also establish that genetic algorithms work better than tabu search for the Turkish day-ahead market. Managerial implications: We deliver a practical tool using innovative optimization techniques to clear the Turkish day-ahead electricity market. We also modify our model to handle similar European day-ahead markets and show that performances of our heuristics are robust under different auction designs.
\end{abstract}

Supplemental Material: The e-companion is available at https://doi.org/10.1287/msom.2018.0767.

Keywords: auctions and mechanism design • day-ahead electricity market $•$ energy-related operations $\cdot$ OM practice

\section{Introduction and Background}

Electricity spot and futures markets play a central role in liberalized electricity markets by promoting price transparency, competition, and risk management (Deng and Oren 2006). Spot markets consist of two separate markets: the day-ahead market (DAM) and the intraday market (IDM). The DAM is the larger of the two and is an auction type of market that determines the dayahead electricity prices for each of the 24 hours of the next day. The second market, the IDM, is much smaller and provides participants with the ability to adjust their day-ahead positions up to a certain amount of time before the physical delivery of electricity (five minutes to two hours). As electricity demand and supply cannot be forecast exactly, there will always be some amount of imbalance on the grid after the IDM is closed for trading, and these imbalances need to be managed. For this purpose, the system operator organizes a last-resort market called the imbalance market (or reserve capacity market) to ensure that supply and demand are equal in real time. The timeline of these markets is illustrated in Figure 1. (See Tanrisever et al. 2015 for a detailed review of the organization and functioning of liberalized electricity markets.)

In electricity market design, the DAM is of central attention because its prices are usually accepted as a reference point for derivative (futures, forwards, swaps, options, etc.) and other bilateral markets. It is also common for regulatory authorities to index electricity tariffs to DAM prices. In this paper, our goal is to outline a DAM we designed for Energy Exchange Istanbul(EXIST) and provide effective solution methods to clear this market, determining electricity prices for the next day. 
There are two main types of DAM designs in liberalized electricity markets: pool- and exchange-type models. In pool-type models, commonly used in U.S. markets (including PJM, ERCOT, and MISO), market participants submit their bids to the market operator and self-schedule their units according to dispatched amounts determined by the operator. There is usually no demand-side participation in pool-type models, and generation companies submit the cost functions of their generating units. In these models, the market operator determines the total cost-minimizing dispatch schedule for the generation units (Derinkuyu et al. 2015). If the resulting market price is below the cost of some generation units operating in the market, then additional side payments are available to those units (Gribik et al. 2007). Real instances of unit commitment and economic dispatch problems generate large-scale optimization problems. Industrial solutions of these problems typically concentrate on heuristics and Lagrangian relaxation methods ( $\mathrm{Li}$ and Shahidehpour 2005, Araoz and Jörnsten 2011, Phan 2012).

In exchange-type models, commonly used in European markets (including NordPool, EPEX, APX, and Belpex), bids are either accepted from the same price (uniform pricing) or rejected, but no side payment is available (linear price regimes). In addition, the demand side is actively involved in day-ahead auctions. Unlike the pool-type models, in exchangetype models, bidders place portfolio-based bids rather than unit-level bids. Turkish DAM is based on the exchange model with some pool-type features, which are explained in more detail in Section 1.2.

\subsection{Day-Ahead Market Organization in Exchange- Type Models}

Participants in DAM auctions include generators, utility companies, large industrial consumers, traders, and electricity retailers. Each participant places a bid to buy or sell a certain amount of electricity at a certain price for the next day. Were the supply and demand functions continuous and divisible, the problem of determining the market-clearing price (MCP) would be trivial. However, in practice, technological and economic constraints necessitate that some bids are placed as indivisible blocks. For example, a coal-powered plant usually operates 24 hours a day, and hence, it places bids to sell electricity as a block for 24 hours. For most coal-powered plants, it is not economical to shut down the plant and start it up again. In another example, natural gas-powered plants only operate for a block of hours during peak times. These block bids introduce complicated integrality requirements to the problem.

The problem of determining the MCP by matching the bidders is complex in many respects. First, this problem does not decompose into 24 separate problems for each hour as bidders place bids that cover multiple hours. In addition, some types of bids have an all-or-nothing property; that is, either the total bid amount is to be accepted, or the bid is not to be accepted at all. Acceptance of some bids may also be linked to the acceptance of other bids. Hence, a combinatorial problem arises. We explain the three most common bid types submitted by market participants in these models.

1.1.1. Hourly Bids. The most common bid type in DAMs is the hourly bid, with which bidders simply specify a list of quantity-price pairs (QPPs) to buy or sell electricity for a single hour of the next day. Let $q$ represent the quantity and $p$ represent the price of a QPP. In a demand hourly bid, the pair $(p, q)$ means that the participant is willing to pay at most $p$ per megawatt hour (MWh) to buy $q$ units of electricity. In a supply hourly bid, the pair $(p, q)$ means that the participant is willing to receive at least $p$ per MWh to sell $q$ units of electricity.

An hourly bid consists of a set of segments defined by consecutive QPPs. A segment in a supply (demand) hourly bid is fully accepted if the MCP of the specific hour is equal to or higher (lower) than the final price of the segment. However, a segment can be partially accepted if the corresponding MCP is between the prices of the consecutive QPPs. In Table 1, we provide a supply hourly bid example and illustrate the matching quantity in Figure 2. By convention, the quantity of electricity to be supplied is given in negative terms.

An hourly bid can be represented as a piecewise linear function generated by linearly interpolating its QPPs. ${ }^{1}$ The matching quantity of an hourly bid is the quantity corresponding to the $\mathrm{MCP}$ on this piecewise linear function. Figure 2 shows the supply curve of the bid given in Table 1. If the MCP is 280 Turkish liras (TL), then the matching quantity becomes $120 \mathrm{MWh}$ for this bid. In this case, the first two segments are fully accepted, and the third one is partially accepted.

Hourly bids are usually placed by hydroelectric, wind, and solar power plants as well as electricity retailers and distributors. Utility companies with a portfolio of generation capacity also place hourly bids to match their generation profile with their demand profile.

1.1.2. Block Bids and Paradoxical Blocks. Block bids are typically placed by coal-and natural gas-powered

Table 1. An Example Hourly Supply Bid

\begin{tabular}{llrrrr}
\hline Hourly bid: & \multicolumn{5}{c}{ Quantity-price pairs } \\
\hline Price (TL/MWh) & 0 & 150 & 200 & 400 & 500 \\
Quantity (MWh) & 0 & -50 & -100 & -150 & -200 \\
\hline
\end{tabular}


Figure 1. Timeline of Electricity Markets

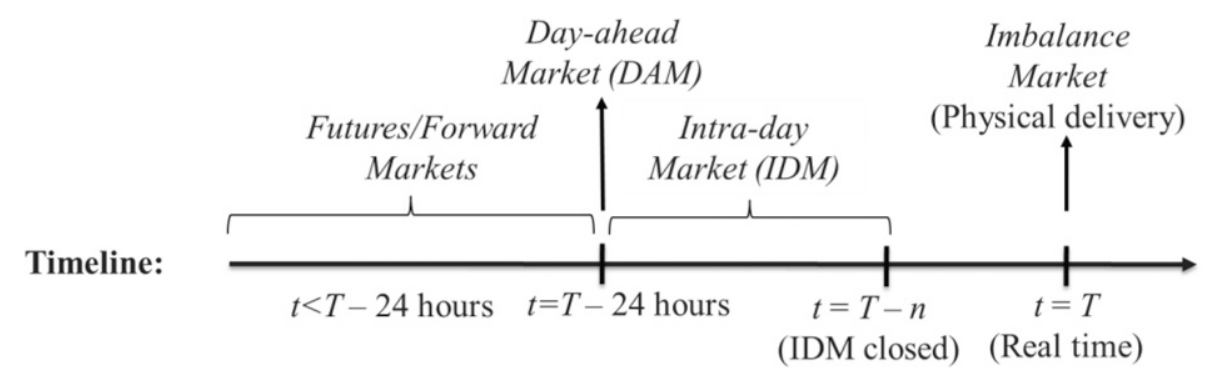

plants as well as by large industrial consumers and electricity retailers. These bids are the second most common type of bid in DAMs and can be viewed as an indivisible set of consecutive hourly bids with a single QPP. In this case, in addition to price and quantity, bidders also specify a consecutive set of hours during which they are willing to trade electricity for the next day. A block bid is accepted or rejected as a full block for all the periods it covers; partial fulfillment of a block bid is not allowed.

In practice, this auction rule to fully accept or reject block bids may result in paradoxical situations. For example, consider the following simple case in Table 2 with one demand block bid and two hourly bids. The demand block bid is to buy $50 \mathrm{MWh}$ at the price of 150 TL/MWh, covering hours 0 and 1 , and the hourly bids are specified as in Table 2.

If we reject the block bid, then the clearing price for both hours ( 0 and 1$)$ is $100 \mathrm{TL} / \mathrm{MWh}$. As the average clearing price is lower than the block bid price, the block bid should be accepted. However, if the block is accepted, then the average clearing price becomes $200 \mathrm{TL} / \mathrm{MWh}$, and this price implies the block should be rejected. This situation is referred to as a paradoxical block because neither accepting nor rejecting the block is feasible. The acceptance condition of a block bid can also be linked to the acceptance of another block bid. Suppose block bid B is linked to block bid A. Then, bid A is called the parent block, and bid $B$ is the child block. If the parent block is rejected, the child block cannot be accepted.
1.1.3. Flexible Bids. These bids specify a single QPP without specifying period information, and they can be accepted at any hour of the day. Similar to block bids, flexible bids have the all-or-nothing property and may also result in paradoxical situations. These bids are typically placed by flexible generation and consumption units, such as storage facilities, dieseland fuel oil-powered plants, and cement producers.

\subsection{Turkish vs. European DAMs}

The daily operations of the Turkish DAM include a number of key steps as summarized in Table 3. In essence, in both Turkey and Europe, a double-sided blind combinatorial auction is used to determine electricity prices for each hour of the next day by matching bidders' supply and demand curves. The two markets mainly deviate from each other in their treatment of paradoxical bids as explained. The Turkish auction allows for paradoxically accepted blocks (PABs); that is, it accepts such blocks. The European auctions allow for paradoxically rejected blocks (PRBs); that is, it rejects such blocks.

In particular, in the Turkish market, a supply (demand) block bid can be accepted even if it is out of the money; that is, the bid price is above (below) the average of the MCPs that the block bid covers. In this case, the corresponding loss incurred by this matching is compensated with a side payment to the participant submitting the block bid. In European auctions, on the other hand, a supply (demand) block bid can be rejected even if it in the money; that is, the

Figure 2. Piecewise Linear Supply Curve Corresponding to the Bid in Table 1

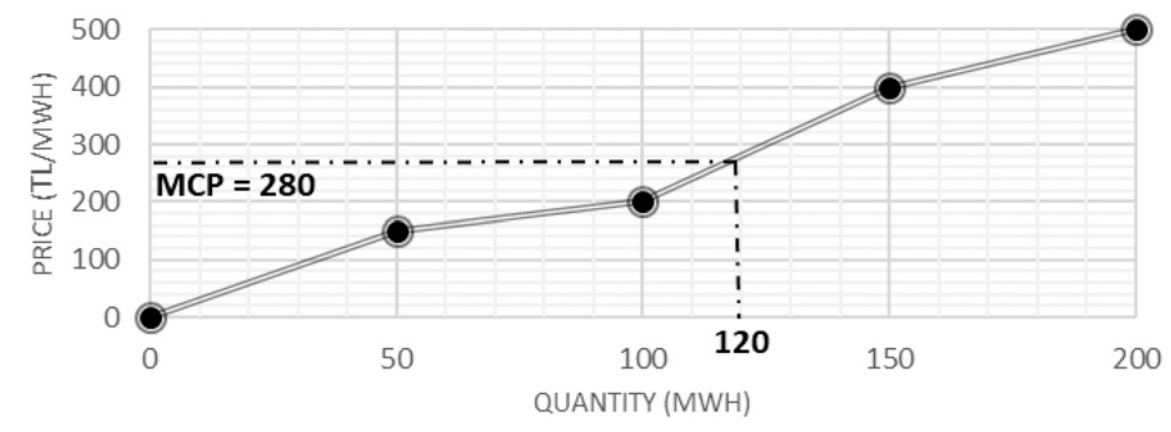


Table 2. An Example of a Paradoxical Block

\begin{tabular}{lrrrrrr}
\hline Hourly bids: & \multicolumn{5}{c}{ Quantity-price pairs } \\
\hline Price (TL/MWh) & 0 & 50 & 100 & 200 & 500 & 1,000 \\
Quantity (for hour 0) & 100 & 75 & 0 & -50 & -100 & -300 \\
Quantity (for hour 1) & 100 & 75 & 0 & -50 & -100 & -300 \\
\hline
\end{tabular}

Block bid: Quantity-price pair

Price (TL/MWh) 150

Quantity (for hours 0 and 1 ) 50

bid price is below (above) the average of the MCPs that the block bid covers, respectively. In this regard, the Turkish DAM is a combination of European exchange models with linear price regimes and U.S. pool models with side payments.

European and Turkish DAMs also differ when accepting and rejecting linked block bids. Unlike in Europe, in Turkish DAMs, parent and child blocks are individually evaluated for acceptance; that is, rejecting the parent block automatically results in rejecting the child block.

\subsection{Contributions}

Considering the integer requirements associated with market participants' bids, paradoxical block bids and the nonlinear social welfare objective, a complicated combinatorial auction arises for the Turkish market operator. In particular, the auction is a nonlinear integer program with as many binary variables as the number of block bids and the number of flexible bids multiplied by the number of periods in a day. In practice, the total number of bids reaches 15,000, and this large problem needs to be solved within minutes every day. Given the practical time limit, solving this problem for optimality with standard optimization packages is not guaranteed for all the possible problem instances; therefore, a heuristic algorithm is needed to quickly obtain high-quality solutions.

Table 3. Daily Operations in the Turkish DAM

\begin{tabular}{|c|c|}
\hline Time slot & Operation \\
\hline $\begin{array}{l}\text { 0000-1700 } \\
\text { hours }\end{array}$ & $\begin{array}{l}\text { Bilateral agreements for the next day are entered } \\
\text { into the system by the market participants. }\end{array}$ \\
\hline $\begin{array}{l}0000-1230 \\
\text { hours }\end{array}$ & $\begin{array}{c}\text { DAM participants submit their bids for the } \\
\text { upcoming day. }\end{array}$ \\
\hline $\begin{array}{l}1230-1300 \\
\text { hours }\end{array}$ & $\begin{array}{l}\text { Collateral payments are checked and bids are } \\
\text { validated automatically. If there is an unusual bid } \\
\text { submission, the market operator has the right to } \\
\text { call the participant for confirmation. }\end{array}$ \\
\hline $\begin{array}{l}1300-1310 \\
\text { hours }\end{array}$ & MCPs are determined by the optimization tool. \\
\hline $\begin{array}{l}1310-1330 \\
\text { hours }\end{array}$ & $\begin{array}{c}\text { Results are published and objections to the bid } \\
\text { matchings are received. }\end{array}$ \\
\hline $\begin{array}{l}1330-1400 \\
\text { hours }\end{array}$ & Objections are evaluated and resolved. \\
\hline 1400 hours & $\begin{array}{c}\text { Finalized MCPs, average hourly prices, and trade } \\
\text { volumes are publicly announced. }\end{array}$ \\
\hline
\end{tabular}

In this paper, we model the DAM auction in Turkey considering the relevant practical constraints. Exploiting the problem structure, we develop innovative adaptations of tabu search (TS) and genetic algorithms (GA) to solve this problem in a reasonable amount of time for practical purposes. Our algorithms have been used by EXIST to clear the Turkish DAM since June 1, 2016. In addition,

- We test the performance of our heuristics and compare them with a commercial solver (CS), which is CPLEX in our case. We show that the Turkish market operator generates significantly more total surplus using our methods. In particular, between June 1, 2016 (when the operator began using our heuristics), and May 31, 2017, our heuristics improved EXIST's total surplus by 448,418 TL per day and 163,672,570 TL per year. This finding corresponds to an improvement of $1.80 \%$ relative to the CS. In addition, the CS failed to generate a feasible solution in $4.10 \%$ of all days, and our heuristics are guaranteed to provide a feasible solution every day.

- We also test the robustness of the value of our heuristics under different auction rules, such as the PRB rule, which is used in European energy exchanges. In this case, the CS failed to generate a feasible solution in $0.55 \%$ of all days within EXIST's specified time limit (10 minutes). Under the PRB rule, although the CS is more effective in providing a feasible solution compared with the PAB case, the quality of such solutions is significantly lower. Therefore, the mean savings provided by our heuristics over the CS are substantially larger for the PRB case. For example, the GA provides a mean savings of 1,986,597 TL per day (7.96\% relative savings).

- The value of our heuristics increases with problem size. We observe that if the problem size doubles, the relative benefit of the GA over the CS reaches $11.92 \%$ under the $\mathrm{PAB}$ rule and $14.35 \%$ under the PRB rule. This amount corresponds to a monthly savings of $89,957,040$ TL and 108,288,600 TL for these cases, respectively.

- We also show that the TS tends to outperform the GA for problem instances in which it is hard to find an initial feasible solution. This result is driven by the feasibility restoration capability of the TS.

- Our algorithms are general enough to be used by other combinatorial auctions, in which bidders place bids for a bundle of items, such as allocating cloudcomputing resources and procuring transportation services. Our modeling of block decisions as binary genes on chromosomes is a convenient and robust approach to handle block bidding behavior and enables our GA to be used by similar combinatorial auctions.

\section{Literature Review}

When goods present complementarities or substitution effects, the joint value of the goods to the bidders can be higher or lower than the sum of the individual value of the goods. In these cases, combinatorial auctions provide a good mechanism by which to capture these 
complementarities and substitution effects for the bidders. Since the seminal paper of Rassenti et al. (1982), combinatorial auctions have been extensively used in practice, ranging from school meal auctions to transportation procurement services (Ledyard et al. 2002, Pekeč and Rothkopf 2003, Sheffi 2004, Chen et al. 2005, Hortaçsu and McAdams 2010, Kastl 2011, Zaman and Grosu 2013, Kim et al. 2014). Cramton et al. (2006) provide a comprehensive survey on this topic.

A typical application area of combinatorial auctions is reverse auctions with package bidding. In these auctions, the buyer allows the bidders to bid in packages of multiple items to benefit from the bidders' economies of scale, scope, or proximity in providing required resources or services. For example, Olivares et al. (2012) examine Chilean school meal auctions, in which suppliers compete to provide daily school meals for multiple geographical areas for a full year. The authors highlight that package bidding may motivate bidders to strategically discount package bids and inflate bid prices for single items, a practice that should be carefully considered when designing combinatorial auctions. Kim et al. (2014) empirically evaluate the performance of combinatorial auctions using the Chilean school meal auction data and find that cost synergies and package discounts play a central role. Similarly, Caplice and Sheffi (2006) study freight transportation service procurement auctions. In their case, carriers can bid for packages of multiple lanes depending on complementarities they have among shipment volumes and routes. Other examples from transportation procurement include London bus route auctions (Cantillon and Pesendorfer 2006) and auctions for truckload shipments for The Home Depot (Elmaghraby and Keskinocak 2003).

In addition to the relatively simple single-round, single-unit combinatorial auctions, there exist very sophisticated models. As giant multinational retail companies have begun to centralize their procurement processes, they have begun to design and run auctions for hundreds of items offered by many suppliers. Famous examples include the strategic sourcing practices of companies such as Mars, Motorola, and P\&G (Hohner et al. 2003, Metty et al. 2005, Sandholm et al. 2006), which have solved the winner determination problem of their sourcing auctions for multiple units, multiple rounds, and multiple attributes. These problems may even include nonprice attributes for items (Sandholm and Suri 2006) and complex bundle discounts (Metty et al. 2005) as well as business rules, such as constraints on the number of winning bidders (Sandholm et al. 2005). The practicability of these complex combinatorial auctions heavily depends on the availability of efficient methods to solve the integer program of the winner determination problem. In this respect, Sandholm et al. (2005) present a problem-specific branch-and-bound algorithm and report improvements compared with CPLEX's integer programming solver, whereas Catalán et al. (2009) suggest a sequencing-based solution method under multiple scenarios. Similarly, Bonomo et al. (2017) propose an efficient polynomial formulation for a multi-item auction under asymmetric bidders and quantity discounts.

The use of combinatorial auctions in electricity markets dates back to the liberalization efforts of these markets in the 1990s. The startup costs of power plants and industrial electricity consumers create complementarities across consecutive operating hours and create motivation for bidders to place bids for a consecutive set of hours to avoid multiple setups (Wolfram 1998, Hortaçsu and Puller 2008, Reguant 2014). Hence, liberalized electricity markets allow some form of complementary bidding, and thus, combinatorial auctions are designed to determine electricity prices (Meeus et al. 2009, Martin et al. 2014, Derinkuyu 2015). Unlike the combinatorial auctions discussed previously, in which the winner receives its bid price, in electricity markets, a single MCP is used to pay market participants. In addition, in electricity combinatorial auctions, there are multiple buyers and sellers placing complementary bids.

One of the first papers in this field, by Meeus et al. (2009), provides a combinatorial auction model to examine the impact of block bid restrictions in electricity markets. The authors' model reflects the auction rules in European markets. For example, as noted, unlike the Turkish market design, European models do not allow for side payments to clear their day-ahead auction markets. Instead, to equalize demand and supply, they allow for rejecting block bids that should be accepted based on the market price, that is, PRBs. Based on their simulation results, the authors argue that exchanges and market participants alike would benefit from relaxing restrictions on block bids.

Martin et al. (2014) consider the objective of maximizing total economic surplus in a market-coupling framework subject to transmission constraints. Their model ensures that no one incurs a loss except for PRBs as in Meeus et al. (2009). They argue that the resulting large-scale optimization problem cannot be solved efficiently by standard solvers and provide an exact algorithm as well as a heuristic, both based on decomposition methods. Madani and Van Vyve (2015) provide a mixed-integer linear program to model European DAM auctions in the presence of stepwise bid curves. Also, for piecewise linear bid curves, they show that the problem can be formulated as a mixedinteger quadratically constrained program with a nonlinear convex quadratic constraint (with integer variables). For both formulations, the authors derive a Benders-like decomposition procedure and strengthen classical Benders infeasibility cuts. 
Derinkuyu (2015) is the first to model the Turkish day-ahead combinatorial auction. He presents a mixedinteger programming formulation and provides aggregation and variable-elimination techniques to significantly reduce the problem size to be solved by commercial solvers within a reasonable amount of time. Unlike the previous papers, Derinkuyu (2015) aims to minimize market prices, which eliminates the nonlinearity with respect to the objective function. In another recent paper, Chatzigiannis et al. (2016) propose an iterative solution procedure based on a mixed-integer programming formulation to solve the current market-coupling problem in European energy markets.

Although there is a growing literature on European electricity DAM auctions, most of this research is mute on the practical use and impact of the suggested algorithms. Although the developed exact methods perform well on real market data, the market operator must find at least one feasible solution in every instance, and this cannot be guaranteed by any exact solution method because the problem is NP-hard. Therefore, we believe that there is a need to develop an algorithm to produce high-quality feasible solutions in a reasonable amount of time for practical purposes. In this paper, we fill this important gap by outlining the development and comparison of the two heuristic methods we devised for EXIST. In addition, unlike most of these papers, our solution methods are used in practice, and we compare their performance against a commercial solver. We provide solid monetary figures on the impact of our solution methods.

\section{The Combinatorial Multiunit Auction Model}

In this section, we model the day-ahead combinatorial auction in the Turkish electricity market with the objective of maximizing social welfare, which is the summation of producer and consumer surpluses. Producer surplus is the difference between the total revenue earned by a producer in return for producing a particular amount of electricity and the bid price for generating this amount of electricity. Consumer surplus is the difference between a consumer's total willingness to pay to consume a particular amount of electricity and the total cost of purchasing this amount of electricity. Figure 3 illustrates these concepts.

Our model includes the bid types that are currently used at EXIST, that is, hourly bids, block bids, and flexible bids. In what follows, we provide the notation and the mathematical model:

\subsection{Sets and Indices}

$>t, \mathrm{~T}$ : time period and set of time periods

$>$ I: set of hourly supply bids

$\triangleright$ J: set of hourly demand bids
Figure 3. Consumer and Producer Surpluses from Hourly Bids

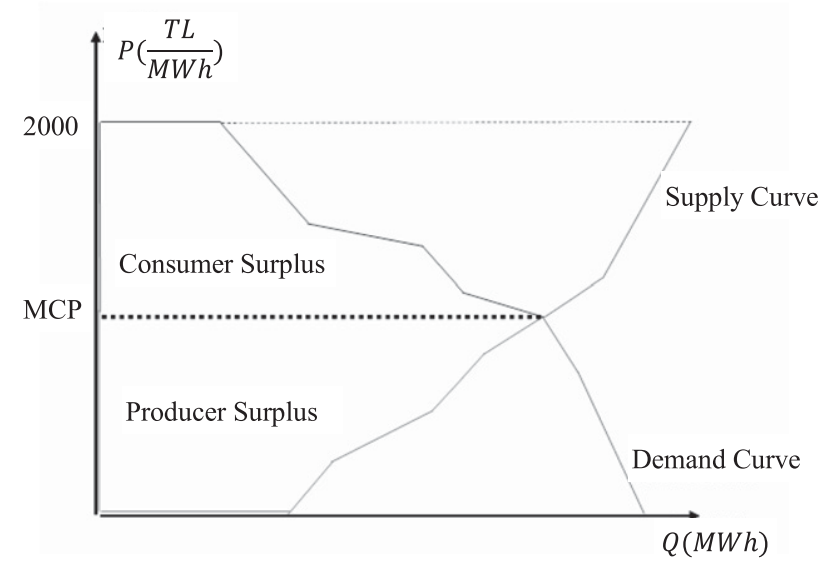

$>l, \mathbf{L}(i)$ : segment index and set of segments for hourly bid $i, i \in \mathbf{I} \cup \mathbf{J}$

$>\mathbf{B}^{\text {s: }}$ : set of supply block bids ( $\mathbf{B}^{\text {sc }}$ : set of child supply block bids)

$>\mathbf{B}^{\mathrm{d}}$ : set of demand block bids ( $\mathbf{B}^{\mathrm{dc}}$ : set of child demand block bids)

$\triangleright \Lambda^{b}$ : set of block bids to which block bid $b$ is linked, $b \in \mathbf{B}^{\mathbf{s}} \cup \mathbf{B}^{\mathbf{d}}$ (all block bids in $\Lambda^{b}$ must be accepted to accept block bid $b$. This set is a singleton.)

$>\mathbf{F}^{\text {s: }}$ set of supply flexible bids

\subsection{Parameters}

$>P_{\min }^{t}$ : lowest possible market-clearing price for period $t$

$>P_{\max }^{t}$ : highest possible market-clearing price for period $t$

$>P_{\min }$ : minimum valid bid price

$>P_{\max }$ : maximum valid bid price

$>P_{i t l}^{0}, P_{i t l}^{1}$ : initial and final prices for segment $l$ of hourly bid $i$ in period $t\left(P_{\min } \leq P_{i t l}^{0}<P_{i t l}^{1} \leq P_{\max }\right.$ for supply bids and $P_{\text {max }} \geq P_{i t l}^{0}>P_{i t l}^{1} \geq P_{\text {min }}$ for demand bids)

$>Q_{i t l}^{0}, Q_{i t l}^{1}$ : initial and final quantity for segment $l$ of hourly bid $i$ in period $t\left(0 \leq Q_{i t l}^{0} \leq Q_{i t l}^{1}\right.$ for all bids $)$

$\triangleright P_{b}, P_{f}$ : price for block bid $b$ and flexible bid $f$

$>Q_{b}, Q_{f}:$ quantity for block bid $b$ and flexible bid $f$

$\gg N_{b}$ : number of time periods that block bid $b$ spans

$>\delta_{b t}$ : binary parameter equal to one if block bid $b$ spans period $t$

$\triangleright \varepsilon$ : a small positive quantity

\subsection{Decision Variables}

$>x_{i t t}$ : accepted fraction of segment $l$ of hourly bid $i$ in period $t, i \in \mathbf{I} \cup \mathbf{J}, t \in \mathbf{T}, l \in \mathbf{L}(i)$

$\triangleright y_{b}$ : one if block bid $b$ is accepted, zero otherwise, $b \in \mathbf{B}^{\mathbf{s}} \cup \mathbf{B}^{\mathbf{d}}$

$\triangleright z_{f t}$ : one if flexible bid $f$ is accepted in period $t$, zero otherwise, $f \in \mathbf{F}^{\mathbf{s}}, t \in \mathbf{T}$

$\triangleright p_{t}$ : market-clearing price in period $t, t \in \mathbf{T}$ 
$>w_{i t l}$ : auxiliary variables to model the bid functions, $i \in \mathbf{I} \cup \mathbf{J}, t \in \mathbf{T}, l \in \mathbf{L}(i)$

\subsection{Day-Ahead Market Clearing Problem with Paradoxically Accepted Blocks (DAM_PAB)}

$$
\begin{aligned}
\operatorname{Max} \mathrm{F}= & \sum_{t \in T} \sum_{i \in J} \sum_{l \in L(i)}\left[0.5^{*} x_{i t l}\left(Q_{i t l}^{1}-Q_{i t l}^{0}\right)\right. \\
& \left.*\left(2 P_{i t l}^{0}+x_{i t l}\left(P_{i t l}^{1}-P_{i t t}^{0}\right)\right)\right] \\
+ & \sum_{b \in B^{d}} N_{b}{ }^{*} Q_{b}{ }^{*} P_{b}{ }^{*} y_{b}+\sum_{f \in F^{d}} Q_{f}{ }^{*} P_{f} * \sum_{t \in T} z_{f t} \\
& -\sum_{t \in T} \sum_{i \in I} \sum_{l \in L(i)}\left[0.5 * x_{i t l}\left(Q_{i t l}^{1}-Q_{i t l}^{0}\right)\right. \\
& \left.*\left(2 P_{i t l}^{0}+x_{i t l}\left(P_{i t l}^{1}-P_{i t t}^{0}\right)\right)\right] \\
- & \sum_{b \in B^{s}} N_{b}{ }^{*} Q_{b}{ }^{*} P_{b} y_{b}-\sum_{f \in F^{s}} Q_{f}^{*} P_{f}^{*} \sum_{t \in T} z_{f t}
\end{aligned}
$$

Subject to

(1) Constraints on supply-demand balance:

$$
\begin{aligned}
\sum_{i \in I} & \sum_{l \in L(i)}\left(Q_{i t l}^{1}-Q_{i t l}^{0}\right) * x_{i t l}+\sum_{b \in B^{s}} \delta_{b t} * Q_{b} * y_{b}+\sum_{f \in F^{s}} Q_{f} * z_{f t} \\
& -\sum_{i \in J} \sum_{l \in L(i)}\left(Q_{i t l}^{1}-Q_{i t l}^{0}\right) * x_{i t l}-\sum_{d \in B^{d}} \delta_{d t} * Q_{d} * y_{d} \\
& -\sum_{g \in F^{d}} Q_{g} * z_{g t}=0, \forall t \in \mathbf{T} .
\end{aligned}
$$

(2) Constraints on hourly bids:

$$
\begin{aligned}
& w_{i t 1} \leq x_{i t 1} \leq 1, \forall i \in \mathbf{I} \cup \mathbf{J}, t \in \mathbf{T} \\
& w_{i t l} \leq x_{i t l} \leq w_{i t(l-1)}, \forall i \in \mathbf{I} \cup \mathbf{J}, t \in \mathbf{T}, l=2, \ldots,|\mathbf{L}(i)|-1 \\
& 0 \leq x_{i t|L(i)|} \leq w_{i t(|L(i)|-1)}, \forall i \in \mathbf{I} \cup \mathbf{J}, t \in \mathbf{T} \\
& p_{t}=P_{\text {min }}^{t}+\sum_{l \in L(i)}\left(P_{i t l}^{1}-P_{i t l}^{0}\right)^{*} x_{i t l}, \forall i \in \mathbf{I}, t \in \mathbf{T} \\
& p_{t}=P_{\text {max }}^{t}+\sum_{l \in L(i)}\left(P_{i t l}^{1}-P_{i t l}^{0}\right)^{*} x_{i t l}, \forall i \in \mathbf{J}, t \in \mathbf{T} .
\end{aligned}
$$

(3) Constraints on block bids (allowing for PABs): Supply (demand) block bids having a bid price less (greater) than or equal to the average MCP of the periods, when the bid is active, must be accepted (except child block bids).

For supply side,

$$
\begin{array}{r}
-N_{b}{ }^{*} P_{b}+\sum_{t \in T} \delta_{b t}{ }^{*} p_{t} \leq\left(-N_{b}{ }^{*} P_{\text {min }}+\sum_{t \in T} \delta_{b t} * P_{\max }^{t}\right) * y_{b}-\varepsilon, \\
\forall b \in \mathbf{B}^{\mathbf{s}} \backslash \mathbf{B}^{\mathrm{sc}} .
\end{array}
$$

For demand side,

$$
\begin{array}{r}
N_{b} * P_{b}-\sum_{t \in T} \delta_{b t} * p_{t} \leq\left(N_{b} * P_{\max }-\sum_{t \in T} \delta_{b t} * P_{\text {min }}^{t}\right) * y_{b}-\varepsilon, \\
\forall b \in \mathbf{B}^{\mathbf{d}} \backslash \mathbf{B}^{\mathrm{dc}} .
\end{array}
$$

(4) Constraints on linked block bids:

$$
y_{b} \leq y_{\lambda}, \forall \lambda \in \Lambda^{b}, b \in \mathbf{B}^{\mathbf{s}} \cup \mathbf{B}^{\mathbf{d}} .
$$

(5) Constraints on flexible bids:

A supply flexible bid must be accepted if the maximum $\mathrm{MCP}$ is equal to or greater than the bid price.

$$
\begin{aligned}
& \sum_{t \in T} z_{f t} \leq 1, \forall f \in \mathbf{F}^{\mathbf{s}} \\
& p_{t}-P_{f} \leq\left(P_{\text {max }}^{t}-P_{\text {min }}\right)^{*} \sum_{t \in T} z_{f t}-\varepsilon, \forall f \in \mathbf{F}^{\mathbf{s}}, t \in \mathbf{T} .
\end{aligned}
$$

(6) Decision variable definitions:

$$
x_{i t l}, p_{t} \in \mathbb{R}^{+}, y_{b}, z_{f t}, w_{i t l} \in\{0,1\} .
$$

In DAM_PAB, we maximize the total surplus of market participants, which is the summation of producer and consumer surpluses. Recall that $p_{t}$ is the MCP in period $t$. Then, the surplus of a producer for each type of bid can be calculated as follows:

For hourly bids (P1),

$$
\begin{gathered}
\left(\sum_{t \in T} \sum_{i \in I} \sum_{l \in L(i)} p_{t}^{*}\left(Q_{i t l}^{1}-Q_{i t l}^{0}\right)^{*} x_{i t l}\right) \\
-\left(\sum _ { t \in T } \sum _ { i \in I } \sum _ { l \in L ( i ) } \left[0.5^{*} x_{i t l}\left(Q_{i t l}^{1}-Q_{i t l}^{0}\right)\right.\right. \\
\left.\left.*\left(2 P_{i t l}^{0}+x_{i t l}\left(P_{i t l}^{1}-P_{i t l}^{0}\right)\right)\right]\right) .
\end{gathered}
$$

In (P1), the first term represents the revenues of a supplier from its hourly bids based on the market price. The second term is the cost of supplying the market. The difference between these two terms is the producer surplus. The surplus term for the block and flexible bids are similarly defined for the producer. As there is a single QPP in the block and flexible bids and they have the all-or-nothing property, the surplus of a supplier is easily calculated by multiplying the bid quantity, number of periods that the bid spans, and the average market-clearing price minus the bid price.

For block bids (P2),

$$
\sum_{b \in B^{s}} y_{b} * Q_{b} *\left(-N_{b} P_{b}+\sum_{t \in T} \delta_{b t} p_{t}\right) \text {. }
$$

For flexible bids (P3),

$$
\sum_{f \in F^{s}} Q_{f} *\left(-P_{f}+\sum_{t \in T} z_{f t} * p_{t}\right) .
$$

As an example, consider the hourly bid given in Table 4.

Table 4. A Partially Accepted Hourly Supply Bid Given that $\mathrm{MCP}=280$

\begin{tabular}{lrrrrl}
\hline Segment & $P^{0}$ & $Q^{0}$ & $P^{1}$ & $Q^{1}$ & $x_{l}^{*}$ \\
\hline 1 & 0 & 0 & 150 & 50 & 1 \\
2 & 150 & 50 & 200 & 100 & 1 \\
3 & 200 & 100 & 400 & 150 & 0.4 \\
4 & 400 & 150 & 500 & 200 & 0 \\
\hline
\end{tabular}


If the MCP is 280 , then the first two segments of the bid are fully accepted. The third segment is interpolated, and $40 \%$ of it is accepted. Correspondingly, the matching quantity is found to be 120 . The revenue collected by this bid is $280 \times 120=33,600$. The cost of generating this quantity is $50 \times 150 / 2+50 \times$ $(150+200) / 2+50 \times 0.4 \times(200+280) / 2=17,300$. So the producer surplus collected by the matching of this bid is $33,600-17,300=16,300$.

Similar to the producer surplus, we can formally define the consumer surplus both for the hourly and block bids as the difference of the total willingness to pay and the associated purchase cost.

For hourly bids (C1),

$$
\begin{aligned}
& \left(\sum_{t \in T} \sum_{i \in J} \sum_{l \in L(i)}\left[0.5^{*} x_{i t l}\left(Q_{i t l}^{1}-Q_{i t l}^{0}\right) *\left(2 P_{i t l}^{0}+x_{i t l}\left(P_{i t l}^{1}-P_{i t l}^{0}\right)\right)\right]\right) \\
& -\left(\sum_{t \in T} \sum_{i \in I} \sum_{l \in L(i)} p_{t}^{*}\left(Q_{i t l}^{1}-Q_{i t l}^{0}\right) * x_{i t l}\right) .
\end{aligned}
$$

For block bids (C2),

$$
\sum_{b \in B^{s}} y_{b} * Q_{b} *\left(N_{b} P_{b}-\sum_{t \in T} \delta_{b t} p_{t}\right) .
$$

For flexible bids (C3),

$$
\sum_{f \in F^{d}} Q_{f} *\left(P_{f}-\sum_{t \in T} z_{f t} * p_{t}\right) .
$$

As the objective function of the DAM_PAB is to maximize the summation of the producer and consumer surpluses, it is equivalent to maximizing P1 + $\mathrm{P} 2+\mathrm{P} 3+\mathrm{C} 1+\mathrm{C} 2+\mathrm{C} 3$. Because of the supply-demand balance constraint and uniform market prices, the total revenue collected by the producers must be equal to the total cost paid by the consumers for the total traded energy in each period of the trading day. Therefore, these two values cancel each other out, and the objective function reduces to the difference of the consumers' total willingness to pay and the producers' cost of generation for the traded energy in the market. Although the cost terms associated with block and flexible bids are linear, there exist quadratic terms in the objective function because of the interpolation of hourly bids. Fortunately, as we show in Proposition 1, the objective function is concave.

The first set of constraints in DAM_PAB ensures that an hourly supply and demand balance is achieved. The second set of constraints limits the matched quantity of an hourly bid with the offered bid quantity. The third constraint set specifies the rule for accepting block bids: supply (demand) block bids having a bid price less (greater) than or equal to the average MCP of the periods, when the bid is active, must be accepted. The fourth constraint set guarantees that a child block cannot be accepted unless its parent is accepted. The fifth set restricts the acceptance of a flexible bid to, at most, one period and also requires that a supply flexible bid must be accepted if the maximum MCP is equal to or greater than the bid price. Finally, the last set of constraints describes the binary and continuous decision variables. The proposed model, DAM_PAB, is a mixed-integer quadratic program with a concave social welfare function, a linear set of constraints, and binary variables associated with block and flexible bids.

Before presenting our solution methods, we first provide two key results about our formulation that significantly reduce the solution efforts. Proposition 1 proves the concavity of the objective function. All proofs are available in Appendix A in the e-companion.

Proposition 1. The objective function $F$ is concave.

Next, the following proposition establishes that, for a given set of block decisions, we do not have to enforce the full acceptance of an hourly bid segment before accepting a succeeding segment in DAM_PAB. This proposition significantly reduces the problem size and helps with the heuristic design process.

Proposition 2. Let $x_{m}^{*}$ and $x_{n}^{*}$ be the optimal values of the accepted fractions of two segments in an hourly bid such that $m<n$ and $q_{m}=Q_{m}^{1}-Q_{m}^{0}, q_{n}=Q_{n}^{1}-Q_{n}^{0}$ are positive. Then, $\left(1-x_{m}^{*}\right) x_{n}^{*}=0$. That is, if $x_{m}^{*}<1$, then $x_{n}^{*}=0$. In addition, if $x_{n}^{*}>0$, then $x_{m}^{*}=1$.

\section{Solution Methods}

In this section, by exploiting the problem structure, we develop two heuristic procedures to determine Turkish DAM prices. The initial phases of both heuristic methods are identical and are based on reducing the size of the problem by aggregating the hourly bids. As we noted in Section 1, an hourly bid is a piecewise linear function, and the matching quantity is the value of this function at the MCP. Instead of separately considering all the hourly bids in a given hour, we can form aggregate supply and demand bids for each hour. In particular, we generate these aggregate piecewise linear curves by adding up all the piecewise linear curves of the hourly bids on the supply and demand sides separately. The resulting curves are nondecreasing and nonincreasing in price for the supply and demand sides, respectively.

Once we aggregate the hourly supply and demand bids, we obtain a single hourly supply and demand bid for each hour of the day. Note that, given a set of block and flexible bid decisions, it is trivial to determine the optimal matching quantities for hourly bids by using Proposition 2. Each accepted block or flexible supply bid shifts the aggregate supply curve to the right by an amount equal to the volume of the accepted bid in relevant periods. Similarly, each 
accepted block or flexible demand bid shifts the aggregate demand curve to the right by an amount equal to the volume of the accepted bid in relevant periods. Finally, the intersection of these curves gives the MCP for each period and the matching quantities for the hourly bids.

Our solution procedure consists of two steps. In the first step, we determine the set of block bids to be accepted to maximize the daily total surplus without considering the flexible bids. We develop two metaheuristics for this purpose: a tabu search and a genetic algorithm. In the second step, given the block bid decisions, we determine which flexible bids are to be accepted. In the next two sections, we present the two metaheuristics specially tailored to the DAM clearing problem.

\subsection{Tabu Search Algorithm}

A TS algorithm is a metaheuristic search method that directs a local search into different areas of the search space. It is well known that local search algorithms may not be able to explore the entire feasible solution space, and they may get stuck at local optima. However, TSs add previously visited solution elements to a list and prohibit their usage to prevent the search from stopping at a local optimum. We used a TS framework tailored to the problem-specific features of the Turkish DAM. As with any TS algorithm, we first define the algorithm design elements: move, tabu law, aspiration criteria, diversification, and stopping condition. We summarize the algorithm elements as follows (see Figure B.1 in the e-companion for a detailed pseudo code of the algorithm).

4.1.1. Move. A move represents the set of changes to a solution to reach its neighbor solution. In our algorithm, we define a move as reversing a block bid decision in a given solution. However, we restrict the set of all possible moves and only allow the following ones because of the combinatorial nature of the problem:

- Rejection of an out-of-the-money block bid

- Acceptance of a block bid

According to this definition, there are $m+n$ different moves for each solution, where $m$ shows the number of accepted out-of-the-money block bids and $n$ shows the number of rejected blocks bids. Each move creates a neighbor of the solution, and by using different moves, we can search the neighborhood of the solution, which is a collection of neighbors. The surplus associated with accepting an out-of-the-money block bid is always nonpositive. Therefore, we expect that the rejection of an out-of-the-money block bid increases the total surplus.

A move may result in an infeasible solution if a parent block bid is rejected while its child block bid is accepted. In the case of infeasibility, we repair the solution by simply rejecting the child and grandchild block bids. This method results in a feasible solution, but it may not be in the neighborhood. Hence, a repair may lead us to a diverse solution.

A solution that gives the best objective among feasible and repaired solutions in the neighborhood is called a candidate solution for the next iteration. At each iteration, a new neighborhood is formed around the candidate solution that was determined at the previous iteration.

4.1.2. Tabu Law. Tabu law determines the rules when a solution is temporarily forbidden from being used as a candidate solution. A solution cannot be a candidate solution when it is on the tabu list, which is a collection of the candidate solutions of the previous iterations. After the neighbors are created around the candidate solution, it is added to the tabu list. A solution that is not on the tabu list can be a candidate solution for the next iterations.

4.1.3. Aspiration Criteria. The aspiration criteria determine the moments when we break the tabu law. If a repaired solution gives the best objective value among the feasible neighbors and repaired solutions but is on the tabu list, then we allow that solution as a candidate solution. For each repaired solution, the aspiration criteria can be used only once for a number of iterations. To count the number of aspirations for each repaired solution, we use an aspiration list.

4.1.4. Diversification. Sometimes local search algorithms spend most of their time searching in a narrow solution space. However, promising solutions may be obtained by a wider exploration of the solution set. In such a case, diversification is needed to cover most of the solution space in a reasonable amount of time. We call our diversification mechanism a jumping procedure. The algorithm jumps to another solution when a maximum number of iterations for a specific solution is reached or the search cannot find a candidate solution for the next iteration in the current neighborhood. Note that the jumping points may be infeasible and need to be repaired. We use four jumping points. In the first one, we accept all supply and demand blocks, which is always feasible. The next three jumping points are the solutions in which we

- accept all supply blocks, reject all demand blocks;

- accept all demand blocks, reject all supply blocks;

- reject all supply and demand blocks.

4.1.5. Stopping Condition. The algorithm stops when the preset time limit is reached. In addition, there is a limit on the number of iterations for a local search around each jumping point. 


\subsection{Genetic Algorithm}

A GA mimics the evolutionary process of natural selection by finding the best solution to a problem among all feasible solutions. The main idea behind this algorithm is that good solutions (good chromosomes) consist of good solution elements (good genes), and merging good elements of solutions results in better solutions. In our context, chromosomes correspond to a sequence of block bid decisions, and genes are individual block bid decisions.

Genetic algorithms are population-based algorithms that try to iteratively improve the quality of the solution population. Starting with an initial population, the algorithm creates new solutions (offspring) from the current population and replaces low-quality solutions in the current population with higher-quality new solutions. At the end, the best solution in the final population is returned. The algorithm stops when a convergence criterion is met or the number of iterations reaches a preset limit. The implementation of the proposed GA consists of the following steps:

(a) Solution representation (coding)

(b) Generation of an initial population

(c) Population update: parent selection, crossover operators, and mutation operators

(d) Stopping conditions

We report on each of these steps in detail for our optimization problem.

4.2.1. Solution Representation (Coding). To determine the sequence of block bids in a solution, we sort the block bids in terms of their so-called moment scores, calculated as follows:

$$
u_{b_{k}}=\left\{\begin{array}{l}
\sum_{t \in T} \delta_{b_{k} t} *\left(P_{b_{k}}-P_{t}^{0}\right) * Q_{b_{k^{\prime}}} \text { for demand blocks } \\
\sum_{t \in T} \delta_{b_{k} t} *\left(P_{t}^{0}-P_{b_{k}}\right) * Q_{b_{k^{\prime}}} \text { for supply blocks, }
\end{array}\right.
$$

where $P_{t}^{0}$ is the MCP in period $t$ found by intersecting only the hourly supply and demand bids, and $k$ is the block index. We sort the block bids in a nonincreasing order of $u_{b_{k}}$ values. Higher values of this score imply higher acceptance chance of the corresponding block bid. This particular sequence of genes in a chromosome allows us to apply the crossover operator in a smarter way, which is explained in Section 4.2.3. We measure the fitness of a solution by the total daily surplus resulting from the vector of MCPs and the set of accepted block bids.

4.2.2. Generating an Initial Population. To start the algorithm, we need an initial population of solutions. The quality and diversity of the solutions in the initial population are important factors in the performance of genetic algorithms. For this purpose, we need to place some high-quality solutions and some diversified solutions into the initial population.

As mentioned earlier, if all bids were hourly bids, then the intersection of the aggregate supply and demand curves for each period would give the corresponding MCPs. Accordingly, we can approximate a problem by transforming block bids to hourly bids for the periods covered by the block bids. Assume we have supply block bid $b$ with a price and quantity pair $\left(P^{b}, Q^{b}\right)$. For each period the block bid covers, we can transform it to an hourly bid by forming the set of QPPs $\left\{\left(P_{\min }, 0\right),\left(P^{b}-\varepsilon, 0\right),\left(P^{b}, Q^{b}\right),\left(P_{\max }, Q^{b}\right)\right\}$, where $\varepsilon$ is a small positive constant.

Now that we have only hourly bids and all the bids can be interpolated, the problem is trivial: form the aggregate supply and demand bids, and their intersection gives us the MCPs. At these prices, some of the block bids may be partially accepted. Rounding the partially accepted block bids, we form a feasible block bid configuration. If this solution violates the link feasibility, we repair the solution and add it to the initial pool.

For population-based heuristics, it is generally suggested to keep diverse solutions in the initial population. For this purpose, we try to generate solutions with different MCPs. We first divide the 24 hours of the day into a number of groups: $g=1,2, \ldots, G$. For each group, we set the MCPs at the lower price limit of the auction, the upper price limit of the auction, or the average of the two limits, resulting in $3^{G}$ different price arrays. For a given price array, we accept and reject the block bids based on the artificially generated average MCPs of the covered periods. After eliminating any duplicate solutions that might exist, we can fill the remainder of the initial pool with these randomly generated solutions.

4.2.3. Population Update. At each iteration of the algorithm, we need to select a subset of solutions (parents) and use this subset (mating pool) to generate new solutions (offspring). We use a crossover operator to create two new solutions from two parent solutions, and we apply a mutation operator to the offspring to improve their quality.

4.2.3.1. Parent Selection for Reproduction. High-quality solutions must be put into the mating pool to generate offspring. For each solution $s$ in the population, we calculate $n_{s}=f_{s} / \bar{f}$, where $f_{s}$ is the total surplus of solution $s$ and $\bar{f}$ is the average total surplus of the population. We put solution $s$ into the mating pool if $n_{s}>0$ so that we favor the selection of high-quality solutions when creating new solutions.

4.2.3.2. Crossover Operator. We randomly select two parents from the current population and apply the 
crossover operator. To cross over the parent solutions, we determine a cut point, from which the solutions are divided into two parts, and then these parts are swapped between each other. We keep the new solutions in a separate list and apply a mutation operator afterward. Before determining a cut point for the crossover, we implement a labeling scheme for the parents based on their gene values. As we describe in Section 4.2.1, the sequence of genes in a chromosome is such that block bids having higher moment scores are placed at the leftmost side of the solution array. Starting from the leftmost gene, we label the genes with an accept decision as high-fit genes, up to the first gene having a reject decision in the sequence. Similarly, starting from the rightmost gene, we label the genes having a reject decision as low-fit genes, up to the first gene having an accept decision. The genes between these two regions are labeled as medium-fit genes. An example for the described labeling scheme is given in Figure 4, in which one corresponds to an accept decision and zero corresponds to a reject decision.

Different solutions will have different labels. However, we expect the gene values in the high- and low-fit parts of different solutions to be very similar and the solutions to differ mostly in the medium-fit area. After labeling the genes, we assign a probability to each gene in the medium-fit region, denoting the chances of each serving as a cut point for the crossover. By doing so, we prevent duplicate offspring solutions. Once we determine the cut point, we swap the parts of the parent solutions. Note that the crossover operation is not guaranteed to produce feasible offspring. We check the feasibility of each offspring and repair it in case of infeasibility.

4.2.3.3. Mutation Operator. We apply a mutation operator to each offspring generated as a way to obtain a better solution in the offspring's neighborhood. For this purpose, we mutate the genes of an offspring one by one as long as we obtain a better feasible solution. This procedure is outlined in Figure B.2 in the e-companion.

4.2.4. Stopping Conditions. At each iteration, we add the offspring solutions to the current population and sort all the solutions in nonincreasing order of their fitness. As we keep the population size fixed, only the best solutions in terms of the fitness score can survive to the next iteration. We continuously monitor the convergence of the population and stop the algorithm when at least one of the following criteria is met:

\begin{abstract}
Criterion 1: For each block bid, calculate the number of times that block bid is accepted in all solutions in the population. Then calculate the acceptance ratio of the block by dividing this number by the population size. If the acceptance ratio of a block bid is either too low or too high, we say that the corresponding gene has converged. If the fraction of converged genes is higher than a threshold value, we stop the algorithm.
\end{abstract}

Criterion 2: If the average fitness value of the solutions in the population is above a preset fraction of the objective function value of the incumbent solution, we stop the algorithm.

Criterion 3: If the number of population updates reaches the iteration limit, we stop the algorithm.

\subsection{Flexible Bids}

After obtaining a solution by applying the algorithms defined in Section 4.2, we now propose an algorithm that adds flexible bids into our solution. We start with the flexible bid with the minimum price and accept it if the bid price is less than the maximum MCP. If the condition holds, the flexible bid is accepted for the period with the maximum MCP, which is then updated. We continue with the next flexible bid with the minimum price and apply the procedure. The algorithm stops if all flexible bids are accepted or the price of the candidate flexible bid is greater than the maximum MCP. This procedure is detailed in Figure B.3 the e-companion.

\section{Computational Results}

In this section, we compare the performance of our algorithms with a CS (CPLEX in our case). The comparisons are based on (i) improvement in total surplus, (ii) computation time, and (iii) the percentage of cases in which a feasible solution is guaranteed. In Section 5.1, we show a comparison with model DAM_PAB, which is the current model used by EXIST. To test the robustness of our results, in Section 5.2, we explore the value of our heuristics for the DAM_PRB case, which

Figure 4. Illustrative Solution Labeling Scheme

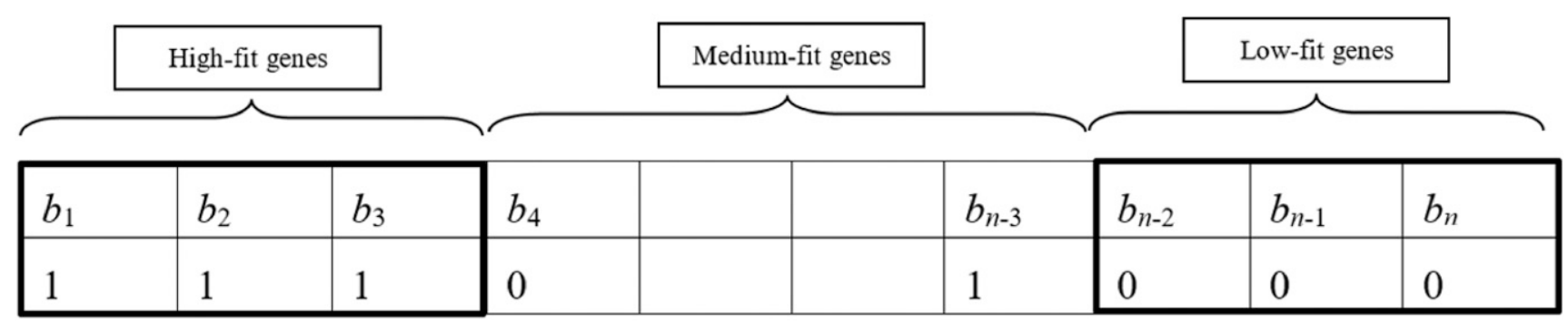


Table 5. The Daily Average Number of Bids in the DAM Auction with Respect to Bid Types Between June 1, 2016, and May 31, 2017

\begin{tabular}{lcccc}
\hline Season & Hourly bid & Supply block bid & Demand block bid & Flexible bid \\
\hline Summer 2016 & 15,196 & 144 & 22 & 2 \\
Fall 2016 & 14,843 & 124 & 20 & 3 \\
Winter 2017 & 14,972 & 90 & 18 & 2 \\
Spring 2017 & 15,955 & 109 & 21 & 1 \\
\hline
\end{tabular}

reflects the forcing constraint rule in Europe (that is, allowing for paradoxically rejected blocks). In addition, in Section 5.3, we test the performance of our heuristics for large problem instances for both the $\mathrm{PAB}$ and PRB cases.

We first provide a descriptive summary of the auction data used in this paper. The data includes 365 real auction instances between June 1, 2016, and May 31, 2017. In Table 5, we report the average numbers of different types of bids submitted daily over this period. The hourly bid is the most commonly used bid type in the market, comprising $98 \%$ of all bids submitted to the system. There are about 135 block bids in the auction, most of which are submitted in the supply side. For flexible bids, we see in Table 5 that they are not preferred by participants and are rarely used. In Table 6, we also present the daily average of offered supply and demand in MWh with respect to bid types. We see that, even though the number of block bids is small, they constitute $20.1 \%$ of the total market volume.

We test our algorithms on a workstation with an Intel ${ }^{\circledR}$ Core $^{\mathrm{TM}} \mathrm{i} 7-4790 \mathrm{CPU}$ with a $3.60 \mathrm{GHz}$ processor with 32 GB RAM and a 64-bit Windows 8.1 Pro operating system. The models and algorithms are coded in Java language using ILOG Java Concert Technology. In the tables, TS and GA refer to the tabu search and genetic algorithm, respectively, and a TS + GA solution denotes the better of the solutions. Additionally, we note that the monetary values in the tables of this section are given in TL (one TL was worth approximately US\$0.286 in June 2017).

\subsection{Numerical Results in the PAB Case}

As we model in Section 3, EXIST allows for PAB bids in day-ahead auctions. In this section, we compare the performance of our heuristics, implemented in practice, with the CS. Table 7 summarizes the results.

Out of 365 days, the CS fails to generate a feasible solution for 15 days within EXIST's specified time limit. In addition, for 73 days, the CS finds a feasible solution but hits the time limit before guaranteeing optimality. The average solution time for the CS is 203 seconds, whereas the same figures for the GA and TS are 14 and 13, respectively. More importantly, both the TS and GA are guaranteed to find feasible solutions within the time limit, which is of crucial importance in operating the energy exchange. ${ }^{2}$

The improvement provided by the GA over the CS ranges between $-10,792 \mathrm{TL}$ per day to $24,909,711 \mathrm{TL}$ per day and averages 443,088 TL per day. Between June 1, 2016, and May 31, 2017, this amount corresponds to a cumulative yearly improvement of $161,727,120 \mathrm{TL}$ in total surplus. In relative terms, the GA improves over the CS by $1.78 \%$. The performance of the TS is lower than the GA and corresponds to a $1.63 \%$ savings over the CS, on average.

We also ran these two algorithms in parallel and selected the best solution (referred to as TS + GA in Table 7). In this case, we observe only a marginal improvement because the GA dominates the TS on almost all days. Finally, the last row of Table 7 presents the case in which our heuristics are integrated with the CS (referred to as TS + GA + CS); that is, the best feasible solution obtained by the TS and the GA is provided to the CS as an initial feasible solution, and the CS runs for 10 minutes in total. This is the configuration used in practice. In this case, the average absolute improvement is about 448,418 TL per day. Between June 1, 2016, and May 31, 2017, this amount corresponds to a cumulative improvement of $163,672,570$ $\mathrm{TL}$ in total surplus. The relative improvement is $1.80 \%$.

Table 6. The Daily Average Volume of Supply and Demand Offered to the DAM with Respect to Bid Types Between June 1, 2016, and May 31, 2017

\begin{tabular}{lcccccc}
\hline & \multicolumn{3}{c}{ Supply (MWh) } & & \multicolumn{2}{c}{ Demand (MWh) } \\
\cline { 2 - 3 } Season & Hourly bid & Block bid & Flexible bid & & Hourly bid & Block bid \\
\hline Summer 2016 & 640,392 & 208,800 & 115 & & 436,488 & 78,144 \\
Fall 2016 & 574,200 & 209,760 & 140 & & 395,568 & 73,608 \\
Winter 2017 & 562,032 & 174,000 & 97 & & 447,216 & 31,896 \\
Spring 2017 & 610,920 & 183,456 & 75 & & 423,312 & 67,056 \\
\hline
\end{tabular}


Table 7. Improvement in Total Surplus by Using Heuristic Algorithms Relative to the CS and Corresponding Solution Times for the PAB Case

\begin{tabular}{|c|c|c|c|c|c|c|c|}
\hline & \multicolumn{3}{|c|}{ Difference from CS (TL) } & \multirow[b]{2}{*}{ Relative mean difference from CS, $\%^{a}$} & \multicolumn{3}{|c|}{ Solution time, s } \\
\hline & Minimum & Mean & Maximum & & Minimum & Mean & Maximum \\
\hline CS & - & - & - & - & 1 & 203 & 600 \\
\hline GA & $-10,792$ & 443,088 & $24,909,711$ & 1.78 & 9 & 14 & 33 \\
\hline TS & $-338,087$ & 407,177 & $24,909,711$ & 1.63 & 5 & 13 & 24 \\
\hline $\mathrm{TS}+\mathrm{GA}$ & $-97,064$ & 444,740 & $24,909,711$ & 1.78 & 9 & 14 & 33 \\
\hline $\mathrm{TS}+\mathrm{GA}+\mathrm{CS}^{\mathrm{b}}$ & $-56,771$ & 448,418 & $24,941,871$ & 1.80 & 14 & 131 & 600 \\
\hline
\end{tabular}

${ }^{a}$ The auction data includes a large number of bids placed with zero price, which is always accepted regardless of the employed solution method. Such bids are placed by renewable generation units, some inflexible coal power plants, and some plants operating on a pass-through contract with the government. Hence, these plants do always operate, and they have a fixed bulk effect on the total surplus. Accordingly, we remove this effect when calculating the relative benefits over the CS. Including such bids into the analysis does not affect the absolute effects reported in the tables, but reduces the relative difference to around $0.15 \%$ in Table 7 because of a denominator effect.

${ }^{\mathrm{b}}$ We have also conducted a set of experiments to consider the case of running pure CPLEX and TS + GA + CPLEX in parallel. The improvements were negligible in this case. In addition, practitioners' concern on transparency and possibility of human mistakes precludes the practical implementation of such a parallel optimization approach.

\subsection{Numerical Results with the PRB Constraints}

In this section, we replace the PAB rule used at EXIST with the PRB rule used in Europe (e.g., by the Euphemia algorithm) and compare the performance of our heuristics with the CS. This experiment enables us to check the robustness of our heuristics' performance relative to different auction designs. The PRB constraints, which replace constraint sets (3) and (5) in the PAB model, are provided.

(3') Constraints on block bids (allowing for PRBs): Demand (supply) block bids having a bid price less (greater) than the average $\mathrm{MCP}$ of the periods in which the bid is active, must be rejected.

For supply side,

$$
\begin{aligned}
& \left(-N_{b} P_{\text {max }}+\sum_{t \in T} \delta_{b t} P_{\text {min }}^{t}\right)\left(1-y_{b}\right) \leq-N_{b} P_{b} \\
& +\sum_{t \in T} \delta_{b t} p_{t}, \forall b \in \mathbf{B}^{\mathbf{s}},
\end{aligned}
$$

and for demand side,

$$
\begin{aligned}
& \left(N_{b} P_{\text {min }}-\sum_{t \in T} \delta_{b t} P_{\text {max }}^{t}\right)\left(1-y_{b}\right) \leq N_{b} P_{b} \\
& -\sum_{t \in T} \delta_{b t} p_{t}, \forall b \in \mathbf{B}^{\mathbf{d}} .
\end{aligned}
$$

(5') Constraints on flexible bids (allowing for PRBs): A supply flexible bid cannot be accepted in a period in which the bid price is greater than the corresponding $\mathrm{MCP}$.

$$
\begin{gathered}
\sum_{t \in T} z_{f t} \leq 1, \forall f \in \mathbf{F}^{\mathbf{s}}, \\
\left(P_{\text {min }}^{t}-P_{\text {max }}\right)\left(1-z_{f t}\right) \leq p_{t}-P_{f}, \forall f \in \mathbf{F}^{\mathbf{s}}, t \in \mathbf{T} .
\end{gathered}
$$

Our results are summarized in Table 8.

Under the PRB rule, out of 365 days, the CS fails to generate a feasible solution for two days within EXIST's 10-minute time limit. However, for 128 days, the CS finds a feasible solution but hits the time limit before guaranteeing optimality. The average solution time for the CS is 289 seconds, whereas the same figures for the GA and TS are 9 and 12 seconds, respectively. In this case, although the CS is more effective in providing a feasible solution, the quality of such solutions is significantly lower compared with the PAB case. Therefore, the mean savings provided by our heuristics over the CS are significantly larger for the PRB case. For example, the GA provides a mean savings of $1,986,597$ TL per day $(7.96 \%$ relative savings) over the CS because, in the PRB case, the CS usually finds a feasible solution quickly by rejecting

\begin{tabular}{|c|c|c|c|c|c|c|c|}
\hline & \multicolumn{3}{|c|}{ Difference from CS (TL) } & \multirow[b]{2}{*}{ Relative mean difference from CS, \% } & \multicolumn{3}{|c|}{ Solution time, s } \\
\hline & Minimum & Mean & Maximum & & Minimum & Mean & Maximum \\
\hline CS & - & - & - & - & 1 & 289 & 600 \\
\hline GA & $-626,962$ & $1,986,597$ & $69,987,943$ & 7.96 & 4 & 9 & 24 \\
\hline TS & $-254,246$ & $2,004,903$ & $69,987,432$ & 8.04 & 2 & 12 & 17 \\
\hline $\mathrm{TS}+\mathrm{GA}$ & $-158,961$ & $2,007,550$ & $69,987,943$ & 8.05 & 4 & 12 & 24 \\
\hline $\mathrm{TS}+\mathrm{GA}+\mathrm{CS}$ & $-16,883$ & $2,012,794$ & $69,987,943$ & 8.07 & 10 & 173 & 600 \\
\hline
\end{tabular}
all blocks. However, improving over this low-quality feasible solution takes a very long time for the CS. In particular, the CS faces a dense branch-and-bound

Table 8. Improvement in Total Surplus by Using Heuristic Algorithms Relative to the CS and Corresponding Solution Times for the PRB Case 
Table 9. Improvement in Total Surplus by Using Heuristic Algorithms Relative to the CS and Corresponding Solution Times for Large Problems for the PAB Case

\begin{tabular}{|c|c|c|c|c|c|c|c|}
\hline & \multicolumn{3}{|c|}{ Difference from CS (TL) } & \multirow[b]{2}{*}{ Relative mean difference from CS, $\%$} & \multicolumn{3}{|c|}{ Solution time, s } \\
\hline & Minimum & Mean & Maximum & & Minimum & Mean & Maximum \\
\hline CS & - & - & - & - & 14 & 351 & 600 \\
\hline GA & $-37,666$ & $2,998,568$ & $25,155,231$ & 11.92 & 26 & 38 & 53 \\
\hline TS & $-461,524$ & $2,823,919$ & $25,002,579$ & 11.22 & 11 & 48 & 76 \\
\hline $\mathrm{TS}+\mathrm{GA}$ & $-37,666$ & $2,998,568$ & $25,155,231$ & 11.92 & 26 & 48 & 76 \\
\hline $\mathrm{TS}+\mathrm{GA}+\mathrm{CS}$ & $-7,769$ & $3,002,939$ & $25,157,643$ & 11.94 & 51 & 273 & 600 \\
\hline
\end{tabular}

tree, in which it is very hard to improve the upper and lower bounds.

We also observe that the TS performs better than the GA under the PRB rule. This is an expected result because our GA is highly specialized for the PAB model used by EXIST. As our design of the TS for the $\mathrm{PAB}$ case is more general, it performs better for the PRB case. Finally, for the TS + GA + CS case, the relative savings reaches $8.07 \%$, which corresponds to a yearly savings of $734,669,810 \mathrm{TL}^{3}$

The TS tends to outperform the GA for problem instances in which it is hard to find an initial feasible solution. The TS searches the optimal solution in both the feasible and infeasible spaces, and the GA focuses on the feasible space only. In its final stage, the TS employs a feasibility restoration algorithm for infeasible solutions. This capability of the TS provides an advantage over the GA. In addition, the GA can be tailored to the PRB case by (i) calibrating the crossover probabilities of the high-, medium-, and low-fit genes on the chromosomes and (ii) calibrating the infeasibility repair probabilities for the chromosomes.

\subsection{Numerical Results for Large Problem Instances}

Historically, we observe that the size of the day-ahead auction is increasing with more market participants and more bids. In this section, we generate large problem instances and test the performance of our heuristics. For this purpose, we combine the auction data of two consecutive days. Hence, the size of the problem roughly doubles. We use auction data from February 1, 2017, to April 1, 2017. Overall, we solve 30 large problems and report the results for the PAB and PRB cases in Tables 9 and 10, respectively.
For both the PAB and PRB cases, the performance of our heuristics substantially increases for large problem instances. For example, the GA saves $11.92 \%$ and $14.35 \%$ over the CS for the PAB and PRB cases, respectively. This amount corresponds to a monthly savings of $89,957,040$ and $108,288,600$ TL for the two cases, respectively. Intuitively, as the problem size grows, the complexity of the problem also increases, and the need for heuristics becomes overwhelming.

Under the PAB case, out of 30 instances, the CS fails to generate a feasible solution for three cases (10\% of all instances) within EXIST's specified time limit. In addition, in 12 cases, the CS hits the time limit before guaranteeing optimality. The average solution time for the CS becomes 351 seconds, whereas the same figures for the GA and TS are 38 and 48 seconds, respectively. Under the PRB case, the CS again fails to generate a feasible solution for three cases and hits the time limit before guaranteeing optimality in 18 cases ( $60 \%$ of all cases). The average solution time for the CS becomes 493 seconds, whereas the same figures for the GA and TS are 22 and 14 seconds, respectively.

In addition, for the PAB and PRB cases, we observe that it becomes harder to solve the problem as (i) the numbers of supply and demand blocks become closer to each other, (ii) block prices become closer to the market-clearing price, and (iii) the proportion of block bids that are valid for daytime hours increases.

\section{Implementation Details}

In this section, we elaborate on the practical implementation of our heuristics at EXIST.

Table 10. Improvement in Total Surplus by Using Heuristic Algorithms Relative to the CS and Corresponding Solution Times for Large Problems for the PRB Case

\begin{tabular}{|c|c|c|c|c|c|c|c|}
\hline & \multicolumn{3}{|c|}{ Difference from CS (TL) } & \multirow[b]{2}{*}{ Relative mean difference from CS, \% } & \multicolumn{3}{|c|}{ Solution time, $\mathrm{s}$} \\
\hline & Minimum & Mean & Maximum & & Minimum & Mean & Maximum \\
\hline CS & - & - & - & - & 12 & 493 & 600 \\
\hline GA & $-528,918$ & $3,609,620$ & $23,683,030$ & 14.35 & 18 & 22 & 30 \\
\hline TS & $-366,782$ & $3,646,955$ & $25,002,579$ & 14.50 & 9 & 14 & 34 \\
\hline $\mathrm{TS}+\mathrm{GA}$ & $-238,496$ & $3,662,118$ & $25,155,231$ & 14.56 & 18 & 22 & 34 \\
\hline $\mathrm{TS}+\mathrm{GA}+\mathrm{CS}$ & $-177,136$ & $3,683,902$ & $25,157,643$ & 14.64 & 42 & 486 & 600 \\
\hline
\end{tabular}




\subsection{Curtailment}

In practice, there is no guarantee that the supply and demand curves will intersect and result in an equilibrium for the day-ahead market. This situation happens under two conditions: (i) when an energy surplus happens at the minimum price and (ii) when an energy deficit happens at the maximum price. If any one of these two cases happens, to restore feasibility, we first remove the block restrictions, that is, constraint set (3) in the optimization model DAM_PAB. After removing these constraints, if there is still no feasible solution (i.e., the curves do not intersect) then hourly bids are uniformly cascaded until a feasible solution is obtained. In these cases, when curtailment happens, the equilibrium price is equal to $0 \mathrm{TL} / \mathrm{MWh}$ (minimum price) or 2,000 TL/MWh (maximum price) for the related hours. We note that curtailment is rare at EXIST and was only observed on seven days between June 1, 2016, and May 31, 2017.

\subsection{Fat-Finger Errors}

A fat-finger error is a term used to describe a keyboard input mistake when entering trades to a computer. EXIST has a three-stage procedure to avoid such errors by market participants:

(a) First, when a market participant places a bid that substantially deviates from its historical bids, an alert is created, and the participant is urged to check the bid.

(b) Second, when bids first enter the EXIST system, an algorithm checks for outliers. Then, experts examine these outliers to see if there may be an error and inform the related market participant.

(c) Third, before the bids are entered into the optimization model, they are automatically checked as to whether they meet the auction criteria.

\subsection{Daylight Saving Time}

During daylight saving changeover days, the auction is adjusted to clear either for 23 or 25 hours.

\subsection{Collateralization}

Market participants are required to provide collateral for their daily trades equal to three times their maximum daily trading volume over the last three months. This method reduces the default risk in the market.

\subsection{Ramp-up and Ramp-down}

There are no ramp-up and ramp-down constraints in the Turkish DAM auction. Hence, market participants that experience imbalances because of rampups and -downs settle these imbalances in the realtime market. Starting from the fourth quarter of 2018, EXIST plans to incorporate such bid types into the day-ahead auction.

\subsection{Objections}

After the day-ahead auction prices are settled and declared, market participants have 30 minutes to object to the results. By law, each objection must be addressed within one hour.

\section{Conclusions}

In liberalized electricity markets, day-ahead market design is of prime importance because prices in this market are usually considered a reference for derivative and other bilateral energy markets. Whereas pool-type DAM models are common in the United States, exchange-type models are used in most European countries. These modeling approaches differ from each other in terms of market clearing, participation of the demand side, dispatching, and unit commitment rules. The Turkish DAM resembles European exchange-type models except for the PAB rule, which is used to handle paradoxical bids. In this paper, we model the day-ahead electricity market auction in Turkey and develop effective tabu search and genetic algorithms to solve this problem in a reasonable amount of time for practical purposes. Overall, the Turkish DAM is a complex combinatorial auction with more than 15,000 bids and a nonlinear welfare objective.

Our algorithms have been used by the Turkish market operator, Energy Exchange Istanbul, since June 1, 2016. We analyze the performance of our heuristics by comparing them with a commercial solver and show that our method improves the total surplus by more than 160 million TL per year. The relative improvement compared with the CS is $1.80 \%$. More importantly, both the TS and GA are guaranteed to find feasible solutions within the time limit. We conclude that the algorithms discussed in this paper significantly improve social welfare compared with the CS. Prior to the implementation of our work, EXIST used to rely on a closed-source algorithm to clear the market without fully considering market needs. Our product not only provides managers with high-quality solutions, but it also enables them to focus on the development of new market rules that can now be easily implemented through our framework.

We also tested the robustness of the value of our heuristics under different auction rules and with larger problem instances. Specifically, we tested our heuristics under the PRB rule used in European energy exchanges. In this case, although the CS is more effective in providing a feasible solution, the quality of such solutions is significantly lower compared with the PAB case. Therefore, the mean savings provided by our heuristics over the CS are significantly larger for the PRB case. For example, the GA provides a mean savings of around 725 million TL per year $(7.96 \%$ relative savings) over the CS because, in the PRB case, the CS usually finds a feasible solution quickly by 
rejecting all blocks. However, improving this lowquality feasible solution takes a very long time for the CS; thus, the performance of our heuristics is significantly larger under the PRB auction rule. In addition, the value of our heuristics increases with the problem size. We observe that if the problem size doubles, the relative benefit of the GA over the CS reaches $11.92 \%$ under the $\mathrm{PAB}$ rule and $14.35 \%$ under the PRB rule. Intuitively, as the problem size grows, the solution effort also increases and the need for heuristics becomes overwhelming.

We observe that it is not possible for commercial solvers to guarantee a feasible solution in combinatorial auctions at EXIST within the time limit. Considering the increasing size and complexity of these auctions over time, efficient heuristics are of crucial importance for energy exchanges under both PRB and $P A B$ rules. The performance of the specific heuristics also depends on the auction parameters and rules. In particular, we note that the TS tends to outperform the GA for problem instances in which it is hard to find an initial feasible solution. This effect is driven by the TS's feasibility restoration algorithm for infeasible solutions. For other cases, the GA typically outperforms the TS.

Combinatorial auctions are commonly used in clearing markets in which the auctioned goods present complementarities or substitution effects; that is, the joint value of the goods to the bidders can be higher or lower than the sum of the individual value of the goods. In these cases, such as allocating cloudcomputing resources and procuring transportation services, block (bundle) and flexible bids are commonly used, and combinatorial auctions provide a good mechanism by which to capture complementarities and substitution effects for the bidders. Our modeling of block decisions as binary genes on chromosomes is a convenient and robust approach to handle blockbidding behavior and enables our GA to be used by similar combinatorial auctions.

Overall, day-ahead market operations provide a fruitful research area. Changes in auction allocation mechanisms and rules may affect bidding behavior in electricity markets, and exploring the impact of such design changes on the total surplus and market prices would be an interesting future research direction. We have also anecdotally observed that supply-anddemand shocks to the electricity market may have diverging effects on bidding behavior, leading to much higher prices during negative supply shocks compared with positive demand shocks. Overall, we believe that a thorough examination of bidding behavior under different auction designs and economic conditions may provide valuable guidance to managers and policy makers when designing such auctions in practice.

\section{Acknowledgments}

All authors have equally contributed to this paper.

\section{Endnotes}

${ }^{1}$ Some European exchanges, such as EPEX and APX, use a stepwise linear function to represent hourly bids.

${ }^{2}$ To measure the optimality of our heuristic solutions, we let CS run up to 24 hours for each problem instance. Then, using the best solution obtained by CS (which is not necessarily optimal), we calculated the upper bounds on the optimality gap of our heuristics as $0.27 \%$ and $0.12 \%$ for the TS and GA, respectively.

${ }^{3}$ We would like to note that European exchanges, such as EPEX, NordPool, and APX, employ the PRB rule and use advanced optimization methods in addition to commercial solvers to clear the market.

\section{References}

Araoz V, Jörnsten K (2011) Semi-Lagrangean approach for price discovery in markets with non-convexities. Eur. J. Oper. Res. 214(2): 411-417.

Bonomo F, Catalán J, Durán G, Epstein R, Guajardo M, Jawtuschenko A, Marenco J (2017) An asymmetric multi-item auction with quantity discounts applied to internet service procurement in Buenos Aires public schools. Ann. Oper. Res. 258(2):569-585.

Cantillon E, Pesendorfer M (2006) Auctioning bus routes: The London experience. Cramton P, Shoham Y, Steinberg R, eds. Combinatorial Auctions (MIT Press, Cambridge, MA), 573-591.

Caplice C, Sheffi Y, Auctions (2006) Combinatorial auctions for truckload transportation. Cramton P, Shoham Y, Steinberg R, eds. Combinatorial Auctions (MIT Press, Cambridge, MA), 539-571.

Catalán J, Epstein R, Guajardo M, Yung D, Martınez C (2009) Solving multiple scenarios in a combinatorial auction. Comput. Oper. Res. 36(10):2752-2758.

Chatzigiannis DI, Dourbois GA, Biskas PN, Bakirtzis AG (2016) European day-ahead electricity market clearing model. Electric Power Systems Res. 140(November):225-239.

Chen RR, Roundy RO, Zhang RQ, Janakiraman G (2005) Efficient auction mechanisms for supply chain procurement. Management Sci. 51(3):467-482.

Cramton P, Shoham Y, Steinberg R (2006) Combinatorial Auctions (MIT Press, Cambridge, MA).

Deng SJ, Oren SS (2006) Electricity derivatives and risk management. Energy 31(6-7):940-953.

Derinkuyu K (2015) On the determination of European day ahead electricity prices: The Turkish case. Eur. J. Oper. Res. 244(3): 980-989.

Derinkuyu K, Tanrisever F, Baytugan F, Sezgin M. (2015) Combinatorial auctions in Turkish day ahead electricity market. Sabuncuoglu I, Kara BY, Bidanda B, eds. Industrial Engineering Applications in Emerging Countries (CRC Press, Boca Raton, FL), 49-64.

Elmaghraby W, Keskinocak P (2003) Combinatorial auctions in procurement. Harrison TP, Lee TH, Neale JJ, eds. The Practice of Supply Chain Management (Springer, New York), 245-258.

Gribik PR, Hogan WW, Pope SL (2007) Market-clearing electricity prices and energy uplift. Report, Harvard Electricity Policy Group, Cambridge, MA.

Hohner G, Rich J, Ng E, Reid G, Davenport A, Kalagnanam J, Lee H, An C (2003) Combinatorial and quantity-discount procurement auctions benefit Mars, Incorporated and its suppliers. Interfaces 33(1):23-35.

Hortaçsu A, Puller SL (2008) Understanding strategic bidding in multi-unit auctions: A case study of the Texas electricity spot market. RAND J. Econom. 39(1):86-114. 
Hortaçsu A, McAdams D (2010) Mechanism choice and strategic bidding in divisible good auctions: An empirical analysis of the Turkish treasury auction market. J. Political Econom. 118(5):833-865.

Kastl J (2011) Discrete bids and empirical inference in divisible good auctions. Rev. Econom. Stud. 78(3):974-1014.

Kim SW, Olivares M, Weintraub GY (2014) Measuring the performance of large-scale combinatorial auctions: A structural estimation approach. Management Sci. 60(5):1180-1201.

Ledyard JO, Olson M, Porter D, Swanson JA, Torma DP (2002) The first use of a combined-value auction for transportation services. Interfaces 32(5):4-12.

Li T, Shahidehpour M (2005) Price-based unit commitment: A case of Lagrangian relaxation vs. mixed integer programming. IEEE Trans. Power Systems 20(4):2015-2025.

Madani M, Van Vyve M (2015) Computationally efficient MIP formulation and algorithms for European day-ahead electricity market auctions. Eur. J. Oper. Res. 242(2):580-593.

Martin A, Müller JC, Pokutta S (2014) Strict linear prices in nonconvex European day-ahead electricity markets. Optim. Methods Software 29(1):189-221.

Meeus L, Verhaegen K, Belmans R (2009) Block order restrictions in combinatorial electric energy auctions. Eur. J. Oper. Res. 196(3): 1202-1206.

Metty T, Raskina O, Schneur R, Kanner J, Potts K, Robbins J (2005) Reinventing the supplier negotiation process at Motorola. Interfaces 35(1):7-23.

Olivares M, Weintraub GY, Epstein R, Yung D (2012) Combinatorial auctions for procurement: An empirical study of the Chilean school meals auction. Management Sci. 58(8):1458-1481.
Pekeč A, Rothkopf MH (2003) Combinatorial auction design. Management Sci. 49(11):1485-1503.

Phan DT (2012) Lagrangian duality and branch-and-bound algorithms for optimal power flow. Oper. Res. 60(2):275-285.

Rassenti SJ, Smith VL, Bulfin RL (1982) A combinatorial auction mechanism for airport time slot allocation. Bell J. Econom. 13(2): 402-417.

Reguant M (2014) Complementary bidding mechanisms and startup costs in electricity markets. Rev. Econom. Stud. 81(4):1708-1742.

Sandholm T, Levine D, Concordia M, Martyn P (2006) Changing the game in strategic sourcing at Procter and Gamble: Expressive competition enabled by optimization. Interfaces 36(1):55-68.

Sandholm T, Subhash S, Andrew G, David L (2005) CABOB: A fast optimal algorithm for winner determination in combinatorial auctions. Management Sci. 51(3):374-390.

Sandholm T, Suri S (2006) Side constraints and non-price attributes in markets. Games Econom. Behav. 55(2):321-330.

Sheffi Y (2004) Combinatorial auctions in the procurement of transportation services. Interfaces 34(4):245-252.

Tanrisever F, Derinkuyu K, Jongen G (2015) Organization and functioning of liberalized electricity markets: An overview of the Dutch market. Renewable Sustainable Energy Rev. 51(November): 1363-1374.

Wolfram CD (1998) Strategic bidding in a multiunit auction: An empirical analysis of bids to supply electricity in England and Wales. RAND J. Econom. 29(4):703-725.

Zaman S, Grosu D (2013) Combinatorial auction-based allocation of virtual machine instances in clouds. J. Parallel Distributed Comput. 73(4):495-508. 